\title{
Animated Pedagogical Agents to Assist Learners and to keep them motivated on Online Learning Environments (LMS or MOOC)
}

\author{
K. Bendou \\ IRF-SIC, Laboratory, IbnZohr \\ B.P.37/S - Agadir - Morocco
}

\author{
El. Megder \\ IRF-SIC, Laboratory, IbnZohr \\ B.P.37/S - Agadir - Morocco
}

\author{
C. Cherkaoui \\ IRF-SIC, Laboratory, IbnZohr \\ B.P.37/S - Agadir - Morocco
}

\begin{abstract}
This paper gives an overview of the first specifications of current research on an animated pedagogical agent to assist learners and to keep them motivate on an online learning environment (LMS or MOOC). It combines characteristics of intelligent agents like: autonomy, ability to perceive, to interact, to reason and to act; and some other characteristics of pedagogical agents as: observing, evaluating, adapting content, recommending, engaging, motivating, etc. The design of this agent is based on a new concept which we have called the Pedagogical Intervention. An intervention may be of different kinds, but it is more precisely used to overcome the current problem of abandonment of learners. We therefore propose to show, through this paper which is a summary of our recent work, the interest and importance of the analysis of the limitations of the online learning environments, in particular the causes of the drop-out problem in order to define adapted pedagogical interventions strategies.
\end{abstract}

\section{Keywords}

Online Learning; Environment; Adaptivity; Recommendation; Feedback; Pedagogical Intervention; Pedagogical agent.

\section{INTRODUCTION}

Over the last decade, the interest in Online Learning Environments (OLE) is growing exponentially. In some ways, this is not a new phenomenon. Researchers working in the online learning sector have been constructing and designing a large amount of models and frameworks which aim to provide teaching or learning to individuals who are distant, but also to those with individual constraints to access to knowledge (spatial, temporal, technological, psychosocial and socioeconomic constraints). As a result, several online learning systems have been developed. Among them we can mention: Adaptive Hypermedia Systems (AHS), Learning Management Systems (LMSs), Virtuel Learning Environments (VLE), Knowledge Management Systems (KMS) and very recently MOOCs [1, 2]. We can also highlight the different ways of learning in informal settings using social networks for learning, discussion forums, blogs, Wikis and access to free resources, etc.

In principle, such environments include course content delivery tools, synchronous and asynchronous activities, exercises and quiz modules, projects, games, workspaces for sharing resources. They also incorporate different types of multimedia learning resources: Videos, Webinars, Podcasts, Apps, etc; but also various nomadic means of access to information: tablets, Smartphone, etc. [3].
These environments, both rich with open varied learning content as well as technologies for interacting and collaborating about this content; offers today new opportunities to learn to each connected resident of the planet [4]. They without hesitation, revolutionize learning.

However, perhaps unsurprisingly, the pervading discourses around the possible contributions of such environments on effective learning point out a number of questions, particularly the issue of dropout.

These are imperative and urgent issues that the OLE community cannot avoid in order to stabilize existing models. If the issue is the construction of OLE that can contribute to a transformation of traditional relationships to knowledge, the learning and teaching required must be achieved under constraints acceptable to the main actors of learning [4]. For the learner, for example, what is sought is a tool that adapts delivery in different dimensions and levels, namely the adaptation of content, presentation, navigation; but also through individual dimensions and/or collaborative, the fun and the massive one [3, 4],

The learner is also looking for tools that allow for a great openness (even massive and therefore very social), and that takes into account their different daily practices. Today's learners also prefer instruction highly connected to their occupations and interests. In this sense, learners from different part of the world can collaborate or interact with global peers and mentors. In other hand, educators in online learning feel desperately an urgent need for automatic ways to motivate and retain online learners. This perspective can be fully integrated into current research on the issue of dropout, motivation and recent solutions implemented through the new concept of adaptive learning.

In the different types of online learning environments, the dropout issue remains the most researched problem over the past ten years. In the particular case of MOOCs, for example, several studies show a large dropout rate estimated at $90 \%$ by most authors of the field [3,9]. The causes of abandonment are related in particular to the lack of motivation and commitment of the learners. Other reasons may be due to occupation, lack of time, isolation, etc. We believe that scripting and nonadaptation of content is a secondary cause. This is also due, in our view to difficulties to keep in OLE a sufficient number of tutors, because of their massive dimension.

The current challenge in OLE is to keep learners motivated [4]. Some authors show that this is possible simply by adding some encouragement phrases above the statement of Mathematics exercises such as "Remember, the more you practice, the more you become intelligent, "or" This could be a difficult problem, but we know that you can get there (do it) ", etc. Learner 
motivation could be improved by adding effective learning strategies. The multiplication of activities and strategies can lead to a large number of possibilities and new instructional interventions. Among these, other authors insist on increasing the number of exercises; adding videos, generating a feedback, asking learners to generate explanations before, during and after the learning process. Other flexibility factors can be obtained simply by increasing the learning time.

All of the points highlighted before lead us to think that a learner who remains isolated, without recommendations, and without pedagogical intervention, can only leads to failure. The advantage of integrating an animated pedagogical agent into MOOC courses seems interesting. In this sense, each learner has a personalized and "humanized" tutor, which makes the human-machine interaction more natural. Animated educational are supposed to play a playful role in guiding, reflecting and interacting with learners. Recent research shows that these characters can support the commitment and motivation of the learners [5]. Other studies point out that students interacting with animated pedagogical agents have been shown to demonstrate deeper learning and higher motivation [6].

In this paper we discuss the contribution of pedagogical agents, which are visible characters in learning environments designed to facilitate learning, to motivate learners and to engage them in an effective learning process. We present in particular an agent, able to propose alternatives and specific intervention strategies. This agent should be able to adapt learning pathways to a learner by proposing pedagogical interventions strategies, according to a number of indicators compiled by the learner model. Before going further into the specifications of the proposed agent, it seems important to go back on a particular OLE, which is the MOOC model. We have chosen this model of e-learning not only because it is new, but in contrast to distance learning platforms (LMSs), has generated new problems related to the notion of distance, which means that most of the learner leaves the course before the end. We note that some problems are almost identical in both the two types of environments, and can lead to common solutions.

In this paper, we first discuss the model of MOOCs with limitations and some improvements in part 2. In the third part, we present the advantages of integrating an animated educational agent into these e-learning environments. The last part will present our first specifications and developments of an animated pedagogical agent that takes into account our approach of improvement of the OLE in order to increase the motivation of learners.

\section{THE DROP-OUT PROBLEM}

In this section, we propose to reconsider the concept of MOOC in order to characterize it while showing the limitations generated and the possible improvements. We are also interested in the reasons for the drop-out problem and the possible solutions based on adaptation strategies and the use of the pedagogical agents.

\subsection{The MOOC Model}

The MOOC model is a major educational innovation generating new ideas and challenges in online education such as massiveness, openness, accessibility, certification, peer assessment, nature and content programming, Etc.

In the MOOC model, we have gone from small groups of learners considered in traditional E-Learning contexts to a massive number reaching thousands of participants. Massiveness creates serious questions about how to manage large heterogeneous groups. The heterogeneity here refers to the nature of the enrolled students, without distinction of prerequisites, diplomas, age, language, etc. This implies in a way, a genuine 'democratization' of access to online resources. The certification produces new economic models [7]. Peer evaluation combined with heterogeneity creates problems of credibility. For example, it is unimaginable that the work of participants can be evaluated by children. Note also that the nature of the contents has also undergone changes using mainly videos programmed over shorter durations [8].

In the literature, we can distinguish two types of MOOCs: xMOOCs and cMOOCs. The xMOOCs take the traditional model of a transmissive approach by considering the teacher as an expert tutor and students as knowledge consumers. The cMOOCs are based on a connectivist approach, which views knowledge as being shared by the different participants, and learning as the process of generating those networks using online and social tools [5].

Despite the fact that there are now several types of MOOCs, most of them have common characteristics. The main shared goal of these learning environments is to allow open access to high quality education, to maintain the process of learning and knowledge creation through social interactions. From a content perspective, they offer a variety of activities, including: reading documents, videos, quizzes, forums, projects, etc. In terms of evaluation, these learning environments are based on formative evaluations instrumented by quizzes, self-assessments built around projects and assignments, and peer reviews as mentioned bellow.

In summary, we simply say that these new potentials of MOOCs have allowed a wide popularity and a shared great acceptance by both researchers, learners as well as other actors [9]. We note however that their future and the potential impact on education are very difficult to expect. We also point out that MOOCs present different limitations generating obstacles and challenges for learners, such us the problem of lack of motivation and engagement which generally leads to the high drop-out rate.

\subsection{Limitations of Traditional MOOCs}

A detailed review of the literature shows that current MOOCs suffer from four main limitations, namely:

\section{- MOOCs Teach to a Certain Percentage of the Learners}

Hill [9] identified different types of MOOC participants: (1) No shows - register, but don't even login, (2) Observers - log in and read content, but do not engage, (3) Drop-Ins - want to achieve a specific goal, which once satisfied, ends the course for them, (4) Passive Participants - consume content, but don't do assignments, (5) Active Participants - fully intend to complete the course and all activities. In this classification, we can immediately see that only the fifth class is likely to complete a course. In addition, latest research demonstrates that among learners who complete courses; most of them seek tangible benefits such as getting a new job, starting a business, or completing prerequisites for an academic program.

\section{- Students Needs Assistance and Immediate Feedback}

Engaging students in the learning process is a big challenge for online learning environments. Designers and teachers must develop appropriate methods of engagement for online education. Research has shown that the higher the levels of interaction in a course, the more students develop positive attitudes towards courses. The feedback, encouragements and assistance are crucial in the success of a Learning Process. The instructor feedback reinforces the course material and 
encourages the students to become more engaged in the learning process. Thus as mentioned before, some authors show that this is possible simply by adding some encouragement sentences above the statement of Mathematics exercises such as "Remember, the more you practice, the more you become intelligent", or "this could be a difficult problem, but we know that you can do it easily", etc. At the same moment, appropriate assistance and help can avoid student disappointment, anxiety, and confusion and learning can be increased. Situations where learners require help and assistance are many; it may be such as help to solve problems, to manage their time, to retrieve best resources, etc.

\section{- Students Wants a great Adaptive instruction}

Even today, most of learning environments are still delivering the same educational content in the same way to learners with different profiles. Everyone nowadays knows that the learners are different, by their needs, expectations, interests, preferences, prerequisites, difficulties, facilities, performance, styles, etc. This difference is generally felt both by learners who are well advanced and at risk of being bored, but also by learners who have problems with their acquisition and are at risk of dropping out. In the context of MOOCs, learners are encouraged to read carefully the resources and participate in activities [11].

However, it is very difficult to track all activities and interactions in these tools because of the massiveness number of enrolled students. What is sought for learners is a tool which adapts its delivery and enhance motivation in different dimensions, ways and levels, namely the adaptation of content, presentation, navigation; but also through individual dimensions and/or collaborative, the fun and the massive one. The learner is also looking for tools that allow for a great openness (even massive and therefore very social), and that takes into account their different daily practices.

\section{- Students Needs Continuous Presence}

In online learning environments, learners are more likely to feel that "someone is there" when needed [12] and in general, they like to enjoy a strong interaction and feel that there is a "human presence" [13].

For Hersh [14], the more the exchanges that occur within an OLE have common features with those that occur in classrooms, the more students will feel connected and engaged in their learning tasks. McCluskey [15] found that the presence of teachers or tutors in an online course is an important factor influencing their success. Above all, he emphasized the presence that manifests itself through the various interventions such as: frequent feedback, clear communications, organizing and maintaining actions, providing students with clear goals, and strong direct instruction, etc.

In open LMS platforms like Moodle, the interface is designed so that teachers can provide lessons and messages using videos recorded with a Webcam. Moodle can also show to tutors and the students who are connected to Skype or to a video chat, in the case they want to have a real-time conversation.

\subsection{The causes of Drop-Out}

As we have been able to show before, the main limitations related to the pedagogical model of MOOCS leads to dropout rates. This is a recurring problem that has resulted in a lot of recent research. El Mhouti [17], presents a synthesis of the literature on the different reasons. He cites the main reasons as follows: no intention to complete, starting late, lack of time, course difficulty, lack of support, lack of digital or learning skills, bad experiences and expectations, peer reviewing, no adaptation is provided. Other authors point out other reasons for abandonment, such as: poor time management, lost rhythm, too difficult course, learning but not doing homework, poor course design [19].

Among the other reasons that have been highlighted in the literature: The difficulty of studying online courses after work, changes in job responsibilities, lack of support from family or employer, lack of feedback on teacher evaluations, lack of Interactions with other participants and teachers.

\section{PEDAGOGICAL AGENTS FOR EFFECTIVE LEARNING IN OLE}

\subsection{Pedagogical Agents in Traditional Learning Environments}

The Intelligent Tutoring System (ITS) is an historical precursor of the new learning environments, with promising results. The ITSs have previously relied on artificial intelligence techniques and had as main objective to simulate the trainer (or the interactions between the learner and the tutor). They constitute the first generation of learning environments which set up pedagogical agents. Pedagogical agents are agents whose function is educational or pedagogical and whose aim is to improve learning.

A profound analysis of these pedagogical agents shows that they are very complex and efficient. We can underline for example, the agent STEVE [22], a personified agent working in a virtual training environment; or BAGHERA [23], which relies on a distributed multi-agent system where each agent can act as tutor, learner-assistant or as a teacher-assistant. Another famous intelligent agent is "AutoTutor" [24], which helps students learn new notions in Newtonian mechanics, computer science, or scientific reasoning through a natural language dialogue that it establishes with learners.

Three types of educational agents have marked the history of ITSs: pedagogical agents, assistant agents and recommendation agents. Without going into the details of the differences between these agents, we will simply say that the main objective of agents is to play different and important roles in a learning environment such us being present and reactive in order to maintain the motivation of learners.

Research suggests that pedagogical agents have the ability to play many roles in the multimedia learning environment, such as demonstrating, scaffolding, coaching, modeling and testing [25]. Animated educational agents are supposed to play a playful role in guiding, reflecting and interacting with learners. Recent research shows that these characters can support the commitment and motivation of the learners [5]. Other studies point out that students interacting with animated pedagogical agents have been shown to demonstrate deeper learning and higher motivation [26, 27].

\subsection{Pedagogical Agents in New Learning Environments (LMSs and MOOCs)}

To our knowledge, little research has been carried out on the integration of pedagogical or recommendations agents in MOOCs and LMSs. As a well known agent in the context of LMS, we mention the agent ABITS (Agent Based Intelligent tutoring system) [28]. Lee and Suh [29] developed an extensible collaborative learning agent that was used to promote interaction among learners. Another example is the one proposed in the work of Lin [30] which have developed several agents communicating with the platform MOODLE using JADE. Other work are in the process of emergence in the context of MOOC learning environments, we so emphasize the work of $[5,11]$. 
Additional research, although independent of LMS and MOOC platforms, claim that Pedagogical Agents foster Engagement, Motivation, and Responsibility [6, 31]. They suppose also that Pedagogical Agents are adaptable and versatile and can Address Learners'Sociocultural Needs. Similar research suggests integrating agents into MOOCs to adapt learning resources to the learner based on his preferences and learning style [17]. Research made in [32] proposes a Recommendation System for MOOCs based on the concept of generating predictions according to other learners' experiences.

Finally, although this research on the integration of agents in OLEs (LMS and MOOC) is rare, we find some attempts almost similar to the ITSs, but with the new vision and the new characteristics of the OLE. Interest is not the least, and the rest of this paper proposes the specifications of a pedagogical agent for these environments based on the concept of educational intervention and other characteristics of agents raised before.

\subsection{Pedagogical Agents VS Drop-Out}

As we presented bellow, the quality of OLE environments depends essentially on their flexibility, the capacity to adapt the delivery and ability to provide feedback and recommendations to maintain students engaged on courses. In that direction, the opportunities of using pedagogical agents in OLE become more and more important. Pedagogical agents are mainly reactive, autonomous and proactive. They can improve interactions and support learning. Indeed, agents have other important characteristics such as ability to perceive, to communicate, reason and act in specialized fields [5].

They also have the ability to cooperate with other agents, which makes them effective in the context of these environments. Research in that domain is not new. Pedagogical agents have already made their proof to simulate collaborative and adaptive behaviors as they appear in some particular works. Different Intelligent Tutoring System (ITS) use agents as virtual entities emulating a human tutor adapting content to the learner's needs, profiles, preferences, rhythm, style [18, 20, 21]. Pedagogical agents are also used as learning aids and recommender agents to adapt content to user profiles [11]. Recent research indicates that agent's can learns from activities and the performance of a user or a group of users, and predict pedagogical decisions and interventions. All of this allows us to make the hypothesis that the agent-approach appears as an interesting technology and natural way to model adaptive feature in OLE.

\subsection{Pedagogical Agents VS Adaptivity}

As we presented bellow, the quality of OLE environments depends essentially on their flexibility, the capacity to adapt the delivery and ability to provide feedback and recommendations to maintain students engaged on courses. In that direction, the opportunities of using pedagogical agents in OLE become more and more important. Pedagogical agents are mainly reactive, autonomous and proactive. They can improve interactions and support learning. Indeed, agents have other important characteristics such as ability to perceive, to communicate, reason and act in specialized fields [5]. They also have the ability to cooperate with other agents, which makes them effective in the context of these environments.

Research in that domain is not new. Pedagogical agents have already made their proof to simulate collaborative and adaptive behaviors as they appear in some particular works. Different ITS systems use agents as virtual entities emulating a human tutor adapting content to the learner's needs, profiles, preferences, rhythm, style $[18,20,21]$. Pedagogical agents are also used as learning aids and recommender agents to adapt content to user profiles [11]. Recent research indicates that agent's can learns from activities and the performance of a user or a group of users, and predict pedagogical decisions and interventions.

All of this allows us to make the hypothesis that the agentapproach appears as an interesting technology and natural way to model adaptive feature in online learning.

\section{THE PAOLE AGENT \\ 4.1 PAOLE PROJECT}

PAOLE (Pedagogical Agent for Online Learning Environments) is a project of the IRF-SIC Laboratory, university IBN ZOHR in Morocco, whose main goal is to examine what agent technologies can bring to the motivation of learners and to minimize the Drop--Out problem.

It is a continuation of the various work carried out on personalization and adaptivity in OLE. In the first development of this Project, a roadmap has been drawn up to take into account a number of constraints, including the fact of taking account agent characteristics in general, but also the characteristics of pedagogical agents. It must also, as we will present next, address the problems highlighted at the beginning of this article on MOOCs.

\subsection{PAOLE Specifications}

A first review of the literature allowed us to distinguish some strategies of the pedagogical intervention of an agent, as we described above. First, we have mentioned four basic principles to be taken into account in MOOCs and OLE in general, namely: (1) the need to take into account the different profiles of learners, (2) continuous presence, (3) assistance and feedback, and (4) adaptation.

Other general strategies can be emphasized, such as: rewarding the effort constantly for failures findings, add frequent and clear comments. We add to this the encouragement of collaboration in forums and social media. It is also necessary to reinforce the observation of traces of the learner and their analysis in order to determine a better intervention strategy. But it goes much better when the intervention is playful and in our case played by an animated agent, which sometimes can distract and consequently distress the learner in failure or in a demotivation state.

The work we present in this section is concerned with an attempt of specifications of an animated pedagogical agent, having the general capacities of the agents and the abilities that we have just described. From an educational point of view, he is classified in the category of a facilitator, but with an additional role of guide and companion. In this context, the pedagogical agent must interact with learners to minimize learner frustration, and enhance learning.

We propose a design based on the concept of pedagogical intervention which we describe in the following. The rest of the paper addresses an area, which to our knowledge is relatively unexplored in the field of OLE. This article draws inspiration from the general importance of the design of the intervention, situating it within the broader landscape of learning analysis, and then examines the specific issues of intervention design for The OLE. In the following, we refer to our agent by the abbreviation PAOLE (Pedagogical Agent for Online Learning Environments).

\subsection{The Pedagogical Intervention}

\subsubsection{Structure of the Pedagogical Intervention}

The pedagogical intervention structure constitutes the main component of PAOLE (Figure 1). Although this notion of intervention is not very common in the literature, we have chose 
it to refer to the different actions of an agent related to the process of help, cooperation, collaboration, adaptation, observation, etc. We note that these actions are not of the same level of intervention and therefore the notion of intervention concerns different levels of learning. Lenoir [33] analyzes this notion in the context of traditional learning, and states that pedagogical intervention is a set of interactions between "the learner, learning objects and the teacher, in connection to the purposes underlying these reports". Following this research, the notion of intervention in PAOLE is the interaction between the learner, the agent and the contents.

Our approach is to determine the importance of the intervention design, placing it in the largest landscape of educational agents. The pedagogical intervention design in this context is concerned with addressing questions such as: why intervene in learning and the teaching process, how should the agent intervene and why (Figure 1).

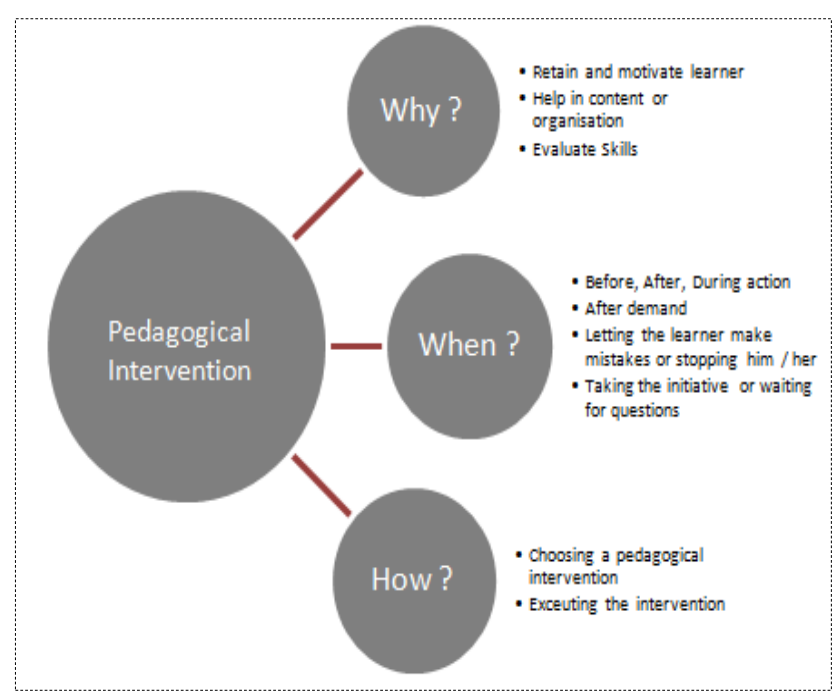

Figure 1. Structure of the Pedagogical Intervention.

To carry out its task, the pedagogical agent PAOLE will have to put in place an effective pedagogical strategy and, if possible, be able to change strategy according to the situation. The pedagogical agent here uses this model to select and adapt his role, his pedagogical strategy, and choose the activities he will put in place. As shown in the figure above, this model allows the answer to three main questions:

- Why: for this question, a pedagogical intervention occurs for several reasons, such as: motivating and retaining a learner, helping him to understand a concept or in order to solve a problem, etc.;

- When: the moment when the pedagogical intervention is carried out is important in learning. Intervention can take place before, during or after the learner's actions, ie the agent must choose the appropriate time either by taking the initiative or responding to the needs and questions of the learner;

- How: the answer to this question involves the selection of the most adapted intervention strategy to execute thereafter among a set of strategies.

\subsubsection{Process of Educational Intervention}

In this section, we present a set of processes that can be used by the agent PAOLE to design pedagogical interventions that support a productive learning (Figure 2).

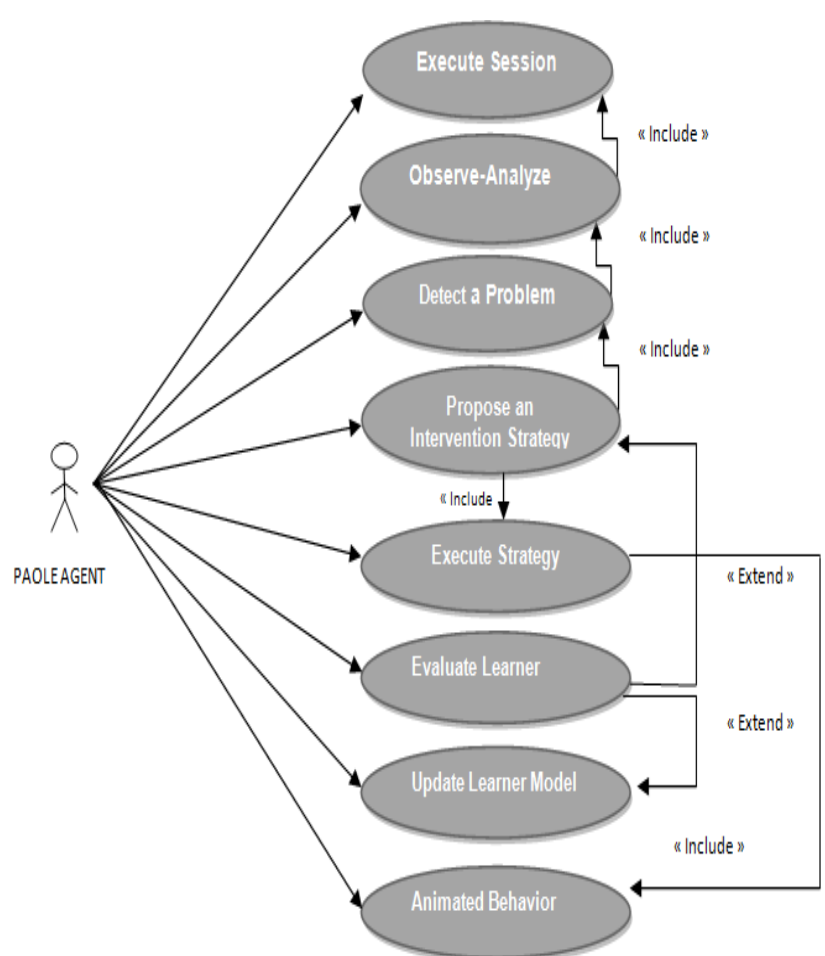

Figure 2. The General Use Case of PAOLE.

There are many indicators which can enable the process to find a strategy appropriate to a given situation. As pointed out in [34], several indicators representing the quantity and quality of a learner's learning activity can be taken into account. This will include, for example, the learner's online presence indicator, the study indicator, the coefficient of the activities carried out and the participation, the results obtained in the activities, the social participation index and the opinion of the tutor. For the coefficient of the activities carried out, this author emphasizes a non exhaustive list, namely: the number of activities carried out (quiz, homework, etc.), number of participation (Wiki, blog, journal, etc.).

Thus, the profile of the user may contain any relevant information relating to him. By taking such information into account, the pedagogical intervention provided may be different for a user who has a high on-line presence coefficient and the user who has a low presence coefficient.

The different processes related to a pedagogical intervention that we have modeled are represented in the figure 2 . We can distinguish six important processes, namely: (i) execute the session, (ii) observe learner, which can lead to analysis and update of the learner profile, (iii) the process of detecting a problem, (vi) the proposal and calculation of intervention strategies, (v) the choice of an adapted intervention and its implementation, and (vi) the operationalization of the strategy in an animated behavior of the agent.

\subsection{Modeling Elements of PAOLE}

\subsubsection{The Architecture of PAOLE}

PAOLE is an autonomous Pedagogical Agent that supports human learning in online Learning Environments with the main objective of keeping them motivated. 


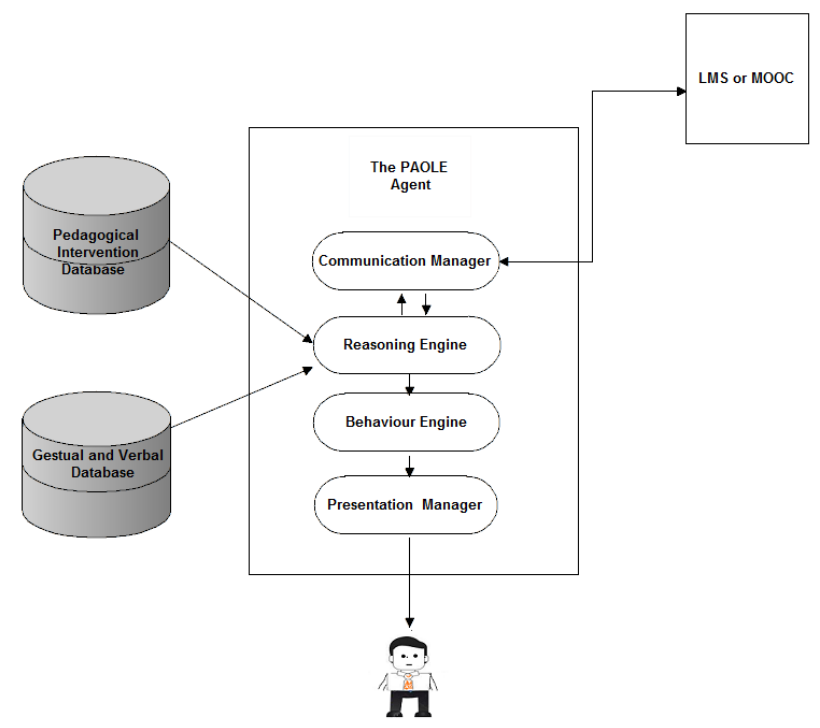

Figure 3. The Architecture of PAOLE.

From a computer architecture perspective, PAOLE consists of four components: (i) a reasoning engine, which monitors student's interactions and generates appropriate pedagogical interventions, (ii) a behavior engine responsible of generating behavior from primitive animations, sounds and speech elements, (iii) a presentation manager, which enables to present generate and present agents' animations, and (iv) a communication module for interactions with the other components of the learning environment as : the learner model and the domain model (see Figure 3). PAOLE is currently under development. It is not created from scratch; we use the interface of the Microsoft agent, which was grafted for the first tests to the platform Moodle.

An animated agent action of PAOLE allows combining several elementary actions of the same character: messages, highlights of components, animations (show a component, applaud, greetings, etc.), and movements on the screen. Other high-level actions based in particular on intervention strategies are represented in the figure 4, in particular the strategies: adaptation, support, engagement and motivation that we present in the following.

\subsubsection{Some Use Cases of PAOLE}

Research suggests that pedagogical agents have the ability to play many roles in the multimedia learning environment, such as demonstrating, scaffolding, coaching, modeling and testing [25].

Research collected in this paper and developed by ourselves and others in the context of pedagogical agents provides further insight into the principles and processes we adopt as we described bellow. In this context, our agent can be characterized by four capabilities that guide its pedagogical intervention (Figure 4). These capabilities are intervention strategies and can be summarized as follows:

- The Adapting strategy: by observing and analyzing the different behaviors and outcomes of the learner, the PAOLE agent can propose different types of adaptations: content, presentation, navigation, etc;

- The Supporting strategy: a learner may at some point need help and support. Two situations are possible: either at the demand of the learner or at the initiative of the agent. The support that can be offered by the agent PAOLE may be at the level of: homework, solving a problem, providing additional resources, modifying the interface, or at another level such as the emotional support;

- The Engaging strategy: the engagement strategy involves engaging a learner in the proposed courses. This strategy will, for example, engage the learner in these courses by offering him varied and advanced content. Involvement can also be at the flexibility of the course agenda by proposing additional weeks to finish homework;

- The Motivating strategy: motivation can be expressed in questions addressed to the learner by the agent, by offering encouragement, providing feedback, encouraging collaboration, encouraging the learner to make other attempts in multiple choice questions, or providing memorable examples (Figure 5).

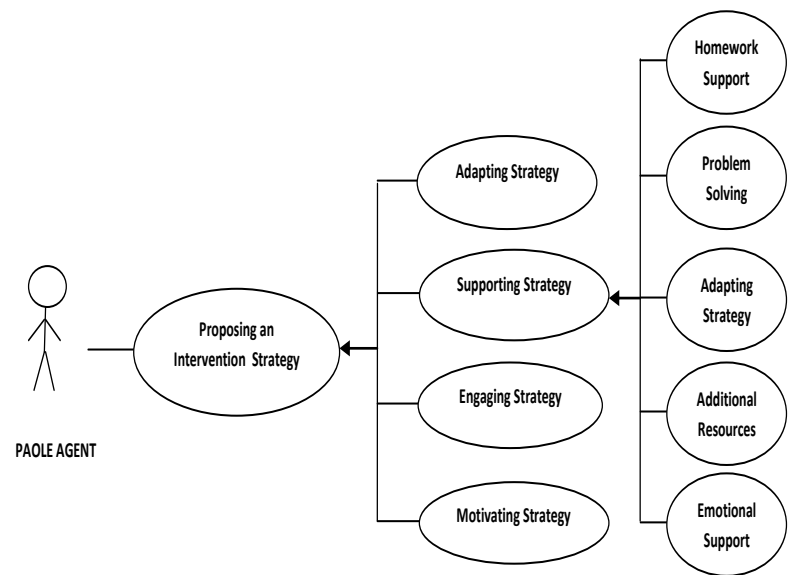

Figure 4. A Use Case of PAOLE Strategies.

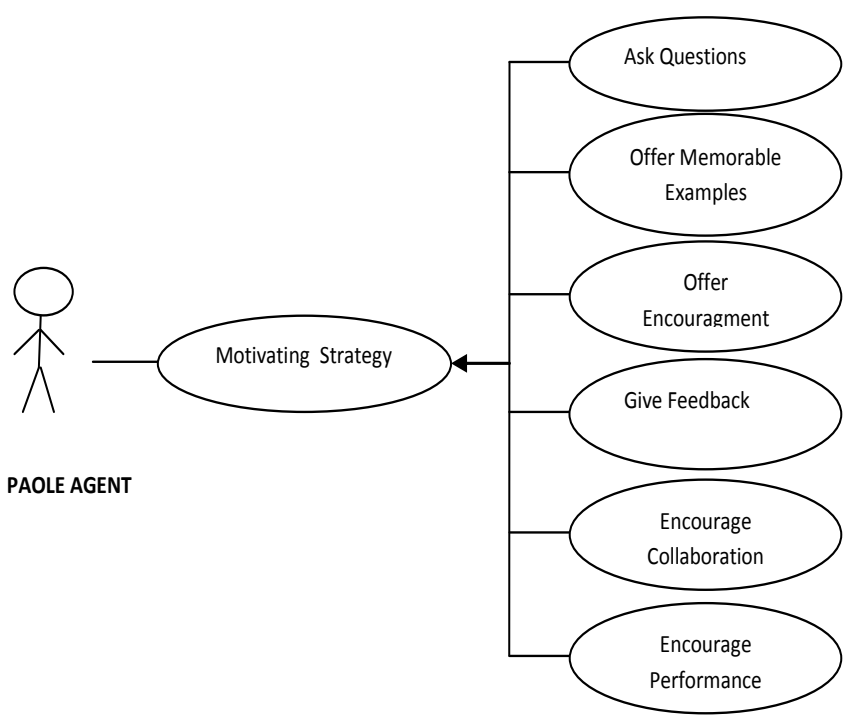

Figure 5. A Use Case of The Motivating Strategy.

\section{CONCLUSION}

Following the various issues presented, and within the limits to be met by this paper, we hope to put a first draft of the current problems related to the issue of the Drop-Out Problem in Online Learning Environments.

After examining the literature, we have given a characterization of the different solutions to keep learners motivated. We first examined the interest of animated pedagogical agents in order to carry out a continuous presence, to propose rapid assistance and feedback, adaptation to the learner model, etc. Other 
strategies for the pedagogical intervention of the PAOLE agent have also been specified, such as: the encouragement of collaboration in forums and social media, the reinforcement of the observation of traces of the learner and its analysis in order to determine a better intervention strategy.

We applied all these elements to give the first specifications of the agent PAOLE, emphasizing the strategies of adaptation, support, commitment and motivation.

Computer architecture of the PAOLE agent is proposed. The communication and the collaboration of the different component generates a behavior that is both animated, but also supports the learner, if at the level of content, at homework, at the organization level, etc. For the development of the agent, we used the interface of the Microsoft agent, which was grafted for the first tests to the Moodle platform, which gave us interesting results which we intend to publish later. In the following, we are developing some of the reasoning component using the JADE platform.

It is clear that several issues remain to be addressed to the expected system. Our work continues along these lines to try to finish a complete and a stable model of the PAOLE agent, which will be tested and validated in a wide spectrum.

\section{REFERENCES}

[1] Wilen-Daugenti, T., (2009). Technology and learning environments in higher education. Peter Lang.

[2] Qazdar, A., Cherkaoui, C., Er-Raha, B. et Mammass, D. (2015). AeLF: Mixing Adaptive Learning System avec système de gestion de l'apprentissage. International Journal of Computer Applications , 119 (15).

[3] Cherkaoui, C., et al. (2015). Un modèle d'adaptation dans les environnements d'apprentissage en ligne (LMS et MOOC). (SITA'2015), 10ème Conférence internationale sur les systèmes Intelligents. IEEE, 2015.

[4] Bonk, CJ, Lee, MM, Reeves, TC et Reynolds, TH eds., (2015). MOOCs and Open Education. Routledge.

[5] Daradoumis, T., Bassi, R., Xhafa, F. and Caballé, S., 2013, October. A review on massive e-learning (MOOC) design, delivery and assessment. In P2P, Parallel, Grid, Cloud and Internet Computing Conference (3PGCIC), IEEE.

[6] Baylor, A. \& Kim, Y. (2003). Validating pedagogical agent roles: Expert, Motivator, and Mentor. In D. Lassner \& C. McNaught (Eds.), Proceedings of EdMedia: World Conference on Educational Media and Technology 2003 (pp. 463-466). (AACE).

[7] Yuan, L., Powell, S. and CETIS, J., (2013). MOOCs and open education: Implications for higher education.

[8] Bakki, A., Oubahssi, L., Cherkaoui, C. et George, S. (2015), Motivation et engagement dans les MOOC: comment accroître la motivation de l'apprentissage en adaptant les scénarios pédagogiques? Dans Design for Teaching and Learning in a Networked World (p. 556559). Springer International Publishing.

[9] Hill, P. (2013). The most thorough summary (to date) of MOOC completion rates, e-Literate, February $26^{\text {th }}$.

[10] Chang, S.H. (2008). Effectiveness of personal interaction in learner - centered paradigm distance education class based on student satisfaction. Journal of Research on Technology in Education . 40 (4).
[11] Zaiane, O. R. (2002, December). Building a recommender agent for e-learning systems. In Computers in Education, 2002. Proceedings. International Conference on (pp. 5559). IEEE.

[12] Galan J-P., Sabadie W. (2001). Evaluation du site Web: une approche par l'expérience de service, 17ème Congrès International de l'Association Française de Marketing, Deauville, 1-26.

[13] Gulz, Agneta. (2004). Benefits of virtual characters in computer based learning environments: Claims and evidence." International Journal of Artificial Intelligence in Education 14.3, 4): 313-334.

[14] Douglas E. Hersh, 2016 - The Human Element https://www.insidehighered.com/news/2010/03/29/lms

[15] McCluskey, F., Kupczynski, L., Ice, P., Wiesenmayer, R. (2010). Student Perceptions of the Relationship between Indicators of Teaching Presence and Success in Online Courses. Journal of Interactive Online Learning, 9, 23-43.

[16] Belanger, Y., \& Thornton, J. (2013). Bioelectricity: A quantitative approach Duke University's first MOOC.

[17] El Mhouti, A., Nasseh, A., \& Erradi, M. (2016). Stimulate Engagement and Motivation in MOOCs Using an Ontologies Based Multi-Agents System. International Journal of Intelligent Systems \& Applications, 8(4).

[18] Stoilescu, D. (2009). Modalities of using learning objects for intelligent agents in learning. International Journal of Doctoral Studies, 4, 49-64.

[19] Nawrot, I. and Doucet, A., (2014). Building engagement for MOOC students: introducing support for time management on online learning platforms. In Proceedings of the 23rd International Conference on World Wide Web (pp. 1077-1082). ACM.

[20] Frasson, C., Mengelle, T., \& Aimeur, E. (1997, August). Using pedagogical agents in a multi-strategic intelligent tutoring system. In Workshop on Pedagogical agents in AI-ED (Vol. 97, pp. 40-47).

[21] Johnson, W. L., Rickel, J. W., \& Lester, J. C. (2000). Animated pedagogical agents: Face-to-face interaction in interactive learning environments. International Journal of Artificial intelligence in education, 11(1), 47-78.

[22] Rickel, J. and Johnson, W.L., (1999). Animated agents for procedural training in virtual reality: Perception, cognition, and motor control. Applied artificial intelligence, 13(4-5), pp.343-382.

[23] Pesty, S., Webber, C. and Balacheff, N., (2001). Baghera: une architecture multi-agents pour l'apprentissage humain. Agents Logiciels, Cooperation, Apprentissage et Activité Humaine ALCAA, pp.204-214.

[24] Graesser, A. C., Lu, S., Jackson, G. T., Mitchell, H. H., Ventura, M., Olney, A., \& Louwerse, M. M. (2004). AutoTutor: A tutor with dialogue in natural language. Behavior Research Methods, 36(2), 180-192.

[25] Clarebout, G., Elen, J., Johnson, W. L., \& Shaw, E. (2002). Animated pedagogical agents: An opportunity to be grasped?. Journal of Educational multimedia and hypermedia, 11(3), 267-286. 
[26] Baylor, A. L., \& Chang, S. (2002). Pedagogical agents as scaffolds: The role of feedback timing, number of agents, and adaptive feedback. In International Conference of the Learning Sciences, Seattle, WA.

[27] Craig, S.D., Driscoll, D.M. and Gholson, B., (2004) Constructing knowledge from dialog in an intelligent tutoring system: Interactive learning, vicarious learning, and pedagogical agents. Journal of Educational Multimedia and Hypermedia, 13(2), p.163.

[28] Capuano, N., Marsella, M. and Salerno, S., (2000) ABITS: An agent based Intelligent Tutoring System for distance learning. In Proceedings of the International Workshop on Adaptive and Intelligent Web-Based Education Systems, ITS

[29] Suh, H. and Lee, S., (2006). Collaborative learning agent for promoting group interaction. ETRI journal, 28(4), pp.461-474.
[30] Lin, F.O., (2010). Integrating JADE agents into MOODLE.

[31] Veletsianos, G. and Russell, G.S., (2013). What do learners and pedagogical agents discuss when given opportunities for open-ended dialogue? Journal of Educational Computing Research, 48(3), pp.381-401.

[32] Garg, V., Tiwari, R. and Gwalior, A.I.(). Hybrid Massive Open Online Course (MOOC) Recommendation System using Machine Learning (2016), In International conference on soft computing techniques in engineering and technology, http://asctet.co.in/papers/OR0033.pdf

[33] Lenoir, Y., (1991). Relations entre interdisciplinarité et intégration des apprentissages dans l'enseignement des programmes d'études du primaire au Québec (Doctoral dissertation, Paris 7).

[34] Bovo, A., (2014). Apprentissage automatique pour l'assistance au suivi d'étudiants en ligne: approche classique et bio-inspirée (Doctoral dissertation). 